\title{
XLI. On the resistance of the electric arc
}

\section{Julius Frith \& Charles Rodgers B.Sc.}

To cite this article: Julius Frith \& Charles Rodgers B.Sc. (1896) XLI. On the resistance of the electric arc , Philosophical Magazine Series 5, 42:258, 407-423, DOI: 10.1080/14786449608620933

To link to this article: http://dx.doi.org/10.1080/14786449608620933

$$
\text { 曲 Published online: } 08 \text { May } 2009 .
$$

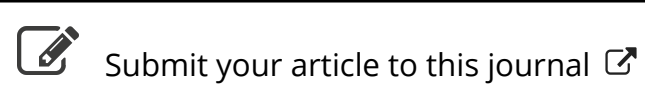

Џلl Article views: 3

Q View related articles 두 
effects from the reflected rays. Thus we introduced in the apparatus in fig. $b$ a lead plate inclined at an angle of $45^{\circ}$ to the axis of the cylinder, and so placed as to reflect the rays through the slit, which was covered with a strip of paper; the arrangement was so sensitive that if the plate had reflected anything like one per cent. of the rays incident upon it, the leak from the metal ring would have been easily detected; there was, however, no trace of a leak. The results of experiments on the photographic effects produced by ray's diffusely reflected from metallic plates seem to show that these rays are fairly abundant. Taking this result in connexion with the absence of any noticeable electrical effect produced by these diffusely reflected rays, we think that the latter differ in character from the incident rays.

We have not been able to detect any effect produced by a magnetic field on the rate of leak ; we tried with the lines of magnetic force parallel and also at right angles to the current, and with both small and saturated currents.

The rate of leak through air that had been dried by standing for three days in the presence of phosphorus pentoxide did not differ appreciably from the damp air of the room.

In conclusion, we desire to thank Mr. E. Everett for the assistance he has given us in these experiments. The period during which a bulb gives ont Röntgen rays at a uniform rate is not a long one, and as most of our experiments required the rate of emission to be constant, they have entailed the use of a very large number of bulbs, all of which have been made by Mr. Everett.

XLI. On the Resistance of the Electric Arc. By JuLius Frith, 1851 Exhibition Scholar, the Owens College, Manchester, and Charles Rodgers, B.Sc., 18.51 Exhibition Scholar, Firth College, Sheffield*.

\section{[Plates III. to V.]}

THERE seems to be some uncertainty as to what is meant by the resistance of the are. Any given are is a phenomenon which exists at a detinite P.D. and current, and any attempt to measure its resistance must alter the state of the arc as little as possible or else we are no longer dealing with the same phenomenon. Hence it seems to us that the only way in which the resistance of the arc can be measured is by the ratio of a very small increment of P.D. applied, to

* Communicated by the Physical Societv : read May 8, 1896. 
<smiles>IC12CNC1C2</smiles> 
the small increment of current produced. It must also be borne in mind that the change in the current must be of so short a duration that the form of the carbons is not in any way altered.

Apparently the only method which fulfils these conditions is one in which a small alternating current is used, superimposed on the main continuous current. This has the effect of rapidly increasing and decreasing by a small amount the current passing through the arc. It seems probable that the effect on the arc of each small increase of current is annulled by the decrease of current immediately following. In fact a comparatively large alternating current may be superimposed on the main continuous current without producing any visible effect on the arc.

We are thus led to define the resistance of the arc as the ratio of a small increment of P.D. applied, to the small increment of current produced. This may be briefly written $\frac{d V}{d A}$. It is most important to distinguish this quantity, which we call the "instantaneous" $\frac{d \mathrm{~V}}{d \mathrm{~A}}$, from the tangent of the inclination of the tangent line of the curve representing the steady values of $\mathrm{V}$ and $\mathrm{A}$, which we will call, for the sake of clearness, the "steady" $\frac{d \mathrm{~V}}{d \mathrm{~A}}$.

We have performed some experiments to exemplify the difference that exists between these two quantities; and also to show that in cases analogous with the arc, where, however, the result can be verified, the instantaneous $\frac{d \mathrm{~V}}{d \mathrm{~A}}$, found by superimposing an alternating current, gives correct values for the resistance.

In one of these experiments a glow-lamp, taking $10 \mathrm{am}$ peres at about 8 volts, was placed in series with three I.E.S. 50 ampere accumulators, and a current sent through against the E.M.F. of the cells. This arrangement is just what is wanted to test the method, namely a resistance in series with a back E.M.F., both of which are functions of the current; and, further, the resistance can be separated from the back E.M.F. and measured, and the result compared with the value cbtained for the resistance by the instantaneous $\frac{d \mathrm{~V}}{d \mathrm{~A}}$.

The result of this experiment is represented on Pl. III. Here are plotted the curves connecting the current and P.D. between the outside terminals of the arrangement and also 
between the terminals of the lamp and of the battery. This last gives the back F.M.F., since the resistance of the cells was small enough to be neglected. The P.D. at the lamp divided by the current gives the resistance; this is plotted on the same diagram ; to make it clearer, however, the scale of ohins is multiplied by 10 . We have measured* the instantaneous $\frac{d \mathrm{~V}}{d \mathrm{~A}}$ at various current strengths, and the values of this are plotted in a dotted line. It is seen that there is a very close agreement between these two measurements of resistance. On the same sheet are plotted values for the steady $\frac{d \mathrm{~V}}{d \mathrm{~A}}$. This differs considerably from both the resistance-curves. This leads us to see that the rapid excursions caused by the alternating current are not along the curve joining the steady values of $V$ and $A$, but along a line which is everywhere more vertical than the tangent to the curve. This line is formed by joining the point on the curve to the instantaneous origin, which is distant from the origin of the diagram by an amount equal to the back E.M.F. at that particular current. Were the electrical excursions to travel along any intermediate path, the value obtained for the instantaneous $\frac{d \mathrm{~V}}{d \mathrm{~A}}$ would be dependent on the frequency. As will be seen later, this is not the case between the experimental limits of 250 and 7 complete alternations per second. If, therefore, the are, as has been affirmed by various authorities, consists of a back F.M.F. and a resistance, we feel justified in applying this method for the measurement of its resistance, which has been found correct in closely analogous cases.

Now at very low frequencies indeed the electrical oscillations would travel along the curve connecting the steady values of $\mathrm{V}$ and $\mathrm{A}$; and this is clearly the meaning of the critical frequency which we have observed with cored carbons (see p. 421), namely, that under the critical frequency the superimposed alternations travel on the steady value curve and become identical with the "steady" $\frac{d V}{d A}$.

Several experimenters have obtained values for the resistance of the arc which agree fairly well amongst themselves, and which seem to show that the are has a positive resistance $\dagger$.

* By method I. below.

+ An abstract of papers bearing on this subject was given by Mrs Ayrton in the 'Electrician,' Sept. 13, 1895. 
It was pointed out, however, by Prof. Ayrton at the Ipswich meeting of the British Association, that although there was a marked agreement between the values obtained by these experimenters, they were not at all in accordance with the conclusions drawn by himself from consideration of the curves obtained by Mrs. Ayrton. These curves connect the P.D. between the carbons with the current passing through the arc, for various fixed lengths of arc, and from them it is seen that for a given arc-length an increase of P.D. is always accompanied by a decrease of current. From this fact Prof. Ayrton concluded that if an attempt were made to measure the resistance of the arc by altering the P.D. between the carbons and finding the corresponding alteration of current produced, the resistance found by taking this ratio must be negative.

This conclusion was strengthened by some experiments made by Mr. Mather at Prof. Ayrton's suggestion. In one of these experiments two points of equal potential were found in a circuit consisting of an arc, a battery, and a resistance. Another battery, consisting of a few cells, of known E.M.F. and resistance was applied between these two equipotential points and the current flowing through the battery was noted. The resistances of the two parallel halves of the circuit, excluding the arc, were known, so that the current which, taking the arc resistance as zero, should flow through this battery could be calculated. Now the value of this calculated current was found to be less than the observed value, no matter in which direction the P.D. was applied, and this result was also obtained when an alternating P.D. was used. Hence the resistance of the arc was apparently less than zero.

The other experiment consisted in running the arc at a steady P.D. and current, suddenly altering the resistance in circuit by a small amount, and noting the changes in the ammeter and voltmeter-readings so produced. The new conditions were maintained only long enough to allow of these readings being takell. 'The arc was then brought back to its former condition before taking another reading. It was found that a change of P.D. in one direction was always accompanied by a change of current in the opposite direction. The results of both experiments were, however, only qualitative.

All these experiments, together with the consideration of the curves found by Mrs. Ayrton, lead to the conclusion that the arc has a negative resistance, while former experimenters had all obtained a positive resistance.

It was in order to throw some light on this discrepancy that we undertook a series of experiments to determine with 
som 3 degree of accuracy the resistance of the arc under various conditions.

\section{Methods.}

A number of methods were tried using alternating currents, of which the following were most successful :-

Method I. is represented diagrammatically in fig. 1. D is

Fig. 1.

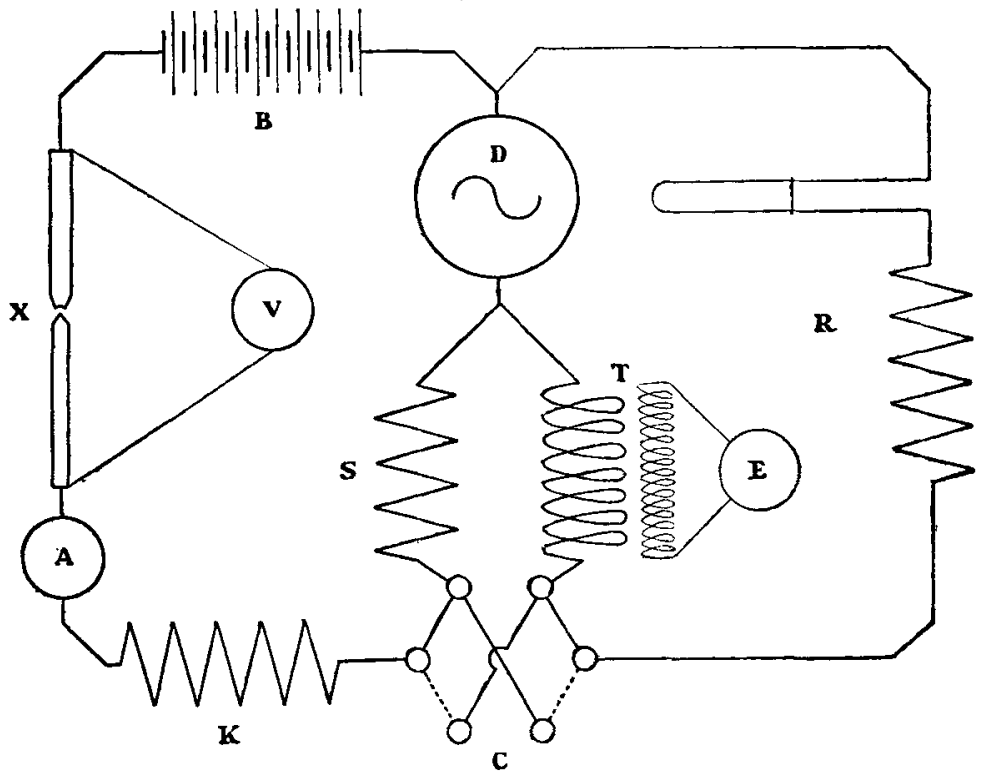

the armature of an alternator, the current from which passes round two circuits in parallel, one of which contains the arc $X$, and the other an adjustable resistance $R$. By adjusting $R$ the alternating currents in the two halves can be made equal. When this is the case the impedances of the two halves to alternating currents must be equal.

In the diagram the continuous-current circuit is shown to the left. It consists of a battery of accumulators $B$, the band-adjusted arc-lamp $\mathrm{X}$, the resistance $\mathrm{K}$, the ammeter $\mathrm{A}$, and (with the commutator $C$ as shown) the resistance $S$ and the alternator D. It will be noticed that the alternator D carries the continuous current, but this of course does not prevent its acting as an alternator.

In order to measure the small alternating current independently of the continuous current flowing we used the airtransformer $\mathrm{T}$, the thick wire coil of which was in series with the alternator $D$, the thin wire coil being connected with an 
electrostatic voltmeter $\mathrm{E}$. The reading of the electrostatie voltmeter is thus unaffected by the continuous current, whilo it is, at a given frequency, a measure of the alternating current flowing.

By means of the commutator $\mathrm{C}$, the air-transformer $\mathrm{T}$ can be thrown into either circuit, the resistance $S$ being by the same operation thrown into the other circuit. The resistance $\mathbf{S}$ is equal to that of the thick wire coil of $\mathrm{T}$, so that when $\mathrm{S}$ replaces $\mathrm{T}$ the continuous current is unaffected by the change.

\section{Metlood of Experimenting.}

The arc was run at the required current and P.D. by altering the number of cells in $\mathrm{B}, \mathrm{K}$ being always kept the same. The current was kept constant by adjusting the arc by hand. Under normal conditions the current could be kept constant to within 1 per cent. $R$ was now adjusted till the deflexion of $\mathrm{E}$ was the same when $\mathrm{T}$ was in either circuit. When balanced, a change of $0.01 \mathrm{ohm}$ in $\mathrm{R}$ caused an appreciable difference in the deflexions of $\mathbf{E}$. It was found to be useless to adjust more accurately than this, since the small variations in quality always found in carbons produced differences of this order in the resistance of the arc.

Let the value of $R$ when a balance is obtained be $R_{1}$. This is equal to the resistance, to alternating currents, of the battery $\left(b_{1}\right)$, the resistance $(k)$, the are lamp and connexions $(l)$, and the arc $(x)$.

$$
\therefore \mathrm{R}_{1}=k+b_{1}+l+x . . \text {. . . . }
$$

The carbons are now firmly screwed together and the number of cells in B reduced till the continuous current is the same as before. $\mathrm{R}$ is again adjusted till the deflexions of $\mathrm{E}$ are equal ; and if $R_{2}$ is the new value thus obtained,

$$
\mathrm{R}_{2}=k+b_{2}+l
$$

$b_{2}$ being the resistance of the portion of the battery now used. The cells are next cut out, the mains leading to them shortcircuited, and a third value $\mathrm{R}_{3}$ obtained,

$$
\mathrm{R}_{3}=k+l \text {. }
$$

From (ii.) and (iii.) we obtain the resistance of $b_{2}$, and by proportion of any number of cells. Putting these values in (i.) we obtain the value of $x$ in ohms.

The advantages of this method are that no calibration of the electrometer is required, the speed of the alternator need not be constant for long periods together, various alternators giving different frequencies and wave-forms can be used without materially altering the circuit, and especially that 
the resistance of the are can be obtained directly in ohms as the difference of two readings of the box $R$.

Method II. is shown diagrammatically in fig. 2 ; the arc

Fig. 2.

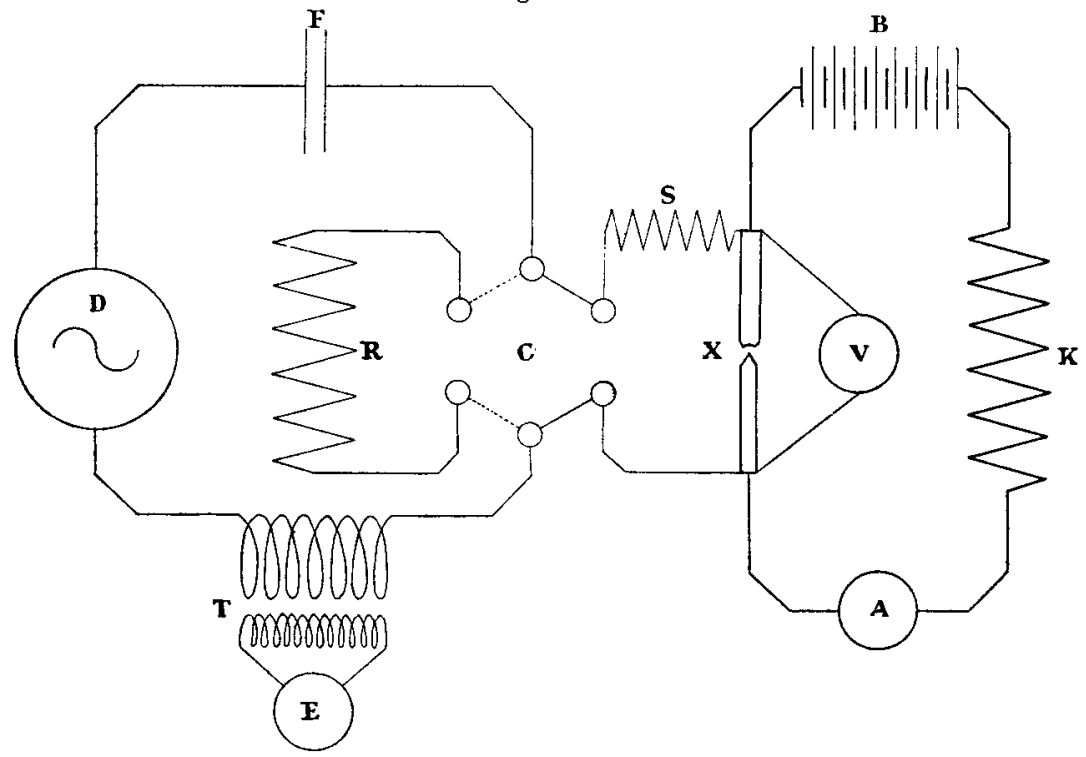

circuit in this case being shown to the right. $\mathrm{X}$ is the arc, $B$ the battery of accumulators, $K$ the resistance, $A$ the ammeter, and $V$ the voltmeter across the arc. $B$ and $K$ were made large, $\mathrm{K}$ being about $11 \mathrm{ohms.}$ The circuit shown on the left consists of the alternator $\mathrm{D}$, the transformer $\mathrm{T}$, which together with $\mathrm{E}$ is now used simply as a delicate alternatecurrent ammeter, a condenser $\mathrm{F}$, and a commutator $\mathrm{C}$. By means of $\mathrm{C}$ we can put in circuit either the resistance $\mathrm{R}$, or the arc-lamp $\mathrm{X}$ in series with the resistance $\mathrm{S}$. The alternating current flowing through $\mathrm{K}$ is negligible compared with that flowing through $\mathrm{X}$ on account of the high resistance of $K$. The condenser $F$ prevents any continuous current due to the P.D. at the arc from passing through the alternator.

\section{Method of Experimenting.}

If $\mathrm{L}$ is the self-induction of the circuit and $\mathrm{F}$ its capacity, the impedance $I$ of the circuit is given by $\sqrt{R^{2}+\left(\frac{1}{F \omega}-L \omega\right)^{2}}$, where $\omega=2 \pi \times$ frequency. This is a minimum when $\mathrm{L} F \omega^{2}=1$. 
The alternator was run at a speed corresponding to this frequency, at which the arrangement is most sensitive to changes of $\mathrm{R}$.

An experiment consists in adjusting $R$ till the deflexions of $\mathrm{E}$ are the same whether $\mathrm{R}$ or $\mathrm{S}+x$ is in circuit. Then

$$
\mathrm{R}=\mathrm{S}+x \text {. }
$$

The experiment was first tried without a resistance at $S$, but it was found impossible when using solid carbons to obtain a balance even when $R$ was zero. In order to make it possible to obtain a balance with $\mathrm{R}$ greater than nothing, a resistance $\mathbf{S}$ numerically greater than the negative resistance of the arc had to be put in series with the arc. In our experiments we kept $\mathbf{S}$ constant and adjusted $R$. The experiment might have been performed by making $R=0$, and adjusting $\mathrm{S}$ till the readings of $\mathrm{E}$ were equal. Then $\mathrm{S}+x$ must be equal to zero, and therefore $x=-S$, or the resistance of the arc is equal to the resistance in the box $\mathbf{S}$ with its sign changed.

This method gives results which agree well with those obtained by Method I.; but on account of the necessity of keeping the speed of the alternator constant the arrangement is more troublesome to manage, and therefore was used only as a check method.

\section{Description of the Apparatus.}

The arc-lamp bad a hand adjustment for each carbon with centering arrangements for the positive. The arc-length was measured by projecting an image of the arc on to a screen by means of a lens in the usual way.

The resistance $K$ consisted of broad platinoid strips bent back on themselves so as to avoid self-induction *. It did not heat appreciably with the largest currents used.

The battery consisted of 50 10-ampere E.P.S. colls arranged in 4 groups of 10 cells each, 4 of 2 , and 2 groups of 1 . These groups could be connected up by means of mercury cups.

The ammeter and voltmeter were Weston instruments of the horizontal type.

The air-transformer $\mathrm{T}$ was made in two sections which were usually used in series. The constants were as follows :-

Section No. 1 :

Fine wire coil.

Diameter of wire (uncovered) $=4 \cdot 3$ mils.

$$
\begin{aligned}
& \begin{aligned}
\text { Number of turns (covered) } & =6 \cdot 8 \\
. . . & =9730 .
\end{aligned} \\
& \text { Resistance . . . . . . }=4707 \text { ohms. }
\end{aligned}
$$

* Described in Phil. Mag. February 1892. 
Thick wire coil.

Size of wire No. 12 S.W.G.

Number of turns . . . . $=128$.

Resistance . . . . . $=0.143$ ohm.

Section No. 2 :

Fine wire coil.

Diameter of wire (uncovered) $=3$ mils.

$\begin{aligned} \text { Number of turns } & \text { (covered) }=5 \\ \text {. . . . } & =29,500 .\end{aligned}$

Resistance . . . . . $=32,120$ ohms.

Thick wire coil.

Size of wire No. 12 S.W.G.

Number of turns . . . . $=\$ 6$.

Resistance . . . . . $=0.112 \mathrm{ohm}$.

The thick wire was double-cotton-covered. The thin wire was silk-covered.

The voltmeter was an Ayrton-Mather reflecting electrostatic voltmeter giving a deflexion of 700 scale-divisions at a distance of 2500 scale-divisions for 100 volts. This instrument was admirably adapted to our purpose on account of its very quick swing and excellent damping.

The resistance $\mathrm{R}$ consisted of $\mathrm{a}$ box wound with thick german-silver wire plugging to $0.1 \mathrm{ohm}$, in series with which was a german-silver wire (No. 10 S.W.G.) doubled back on itself and carrying a slider. Points were marked out at distances corresponding to $0.01 \mathrm{ohm}$. These spaces were about 10.7 centims. long.

The Alternator. Several different alternators were used. The one used while obtaining the curves given was a Pyke and Harris machine, which was especially suitable, since it had no sliding contacts, both armature and field being fixed. The other machines were-tor high frequencies a Ferranti, and for low frequencies a Gramme alternator. The fields in all cases were excited by storage-cells.

The condenser had usually a capacity of about 60 microfarads.

\section{Variation of Conditions.}

The conditions of the experiments were varied as much as possible. We have studied the effect on the resistance of the urc of variations in the amount, frequency, and wave-form of the alternating current; the effect of different kinds of c.rbons and different P.Ds. and currents ; the effect of using different combinations of cored and solid carbons, of carbons cored with substances other than carbon; and the effect of the relative size of the carbons. 


\section{Results.}

The amount of alternating current between the limits we have used does not appear to have any influence on the value obtained for the resistance of the arc. The largest alternating current used had a R.M.S. (root mean square) value equal to about 10 per cent. of the continuous current. The smallest had about one-tenth of this value. In obtaining the curves shown, the R.M.S. of the alternating current was usually about 0.5 ampere.

The frequency also did not have any effect on the resistance of the arc between the limits 250 and $\tau$ complete alternations per second. Frequencies above 150 were obtained from a Ferranti alternator; those between 150 and 45 from a Pyke and Harris; and those between 24 and 7 from a Gramme machine.

Besides these three machines a Mordey transformer of ratio 1:1 was sometimes used, with its primary in circuit with the Pyke and Harris alternator. On account of its having a closed iron circuit it was not so suitable, but it gave the same results as the alternators.

We have thus obtained considerable varietr in the ware form as well as in the frequency. Hence the wave form does not affect our measurements of the resistance of the arc.

The values thus obtained for the resistance of the arc under various conditions are plotted in Pls. IV. \& V. Pl. IV. shows the relation between the resistance of the arc and the current, the P.D. between the carbons being kept constant. Pl.V. fig. 1 shows the relation between the resistance of the arc and the P.1). between the carbons, the current being kept constant. Pl.V. fig. 2 shows the relation between the resistance of the are and the are length, the current being kept constant. In all these cases the resistance is measured from the central line marked 0 , positive values ahove the line and negative values below the line.

Certain makes of carbon have been selected for experiment, namely, Apostle, Brush, Thomson-Houston, and Carré carbons, as representative of the various qualities of earbon now in use commercially.

The diameter of the positive carbon was in all cases 11 millim., the diameter of the negative was 9 millim. in the case of the Apostle and Brush carbons, 8 millim. in the case of the Carré carbons, and 11 millim. in the case of the Thomson-Houston carbons. These sizes were selected as being most suitable for the currents we were able to take 

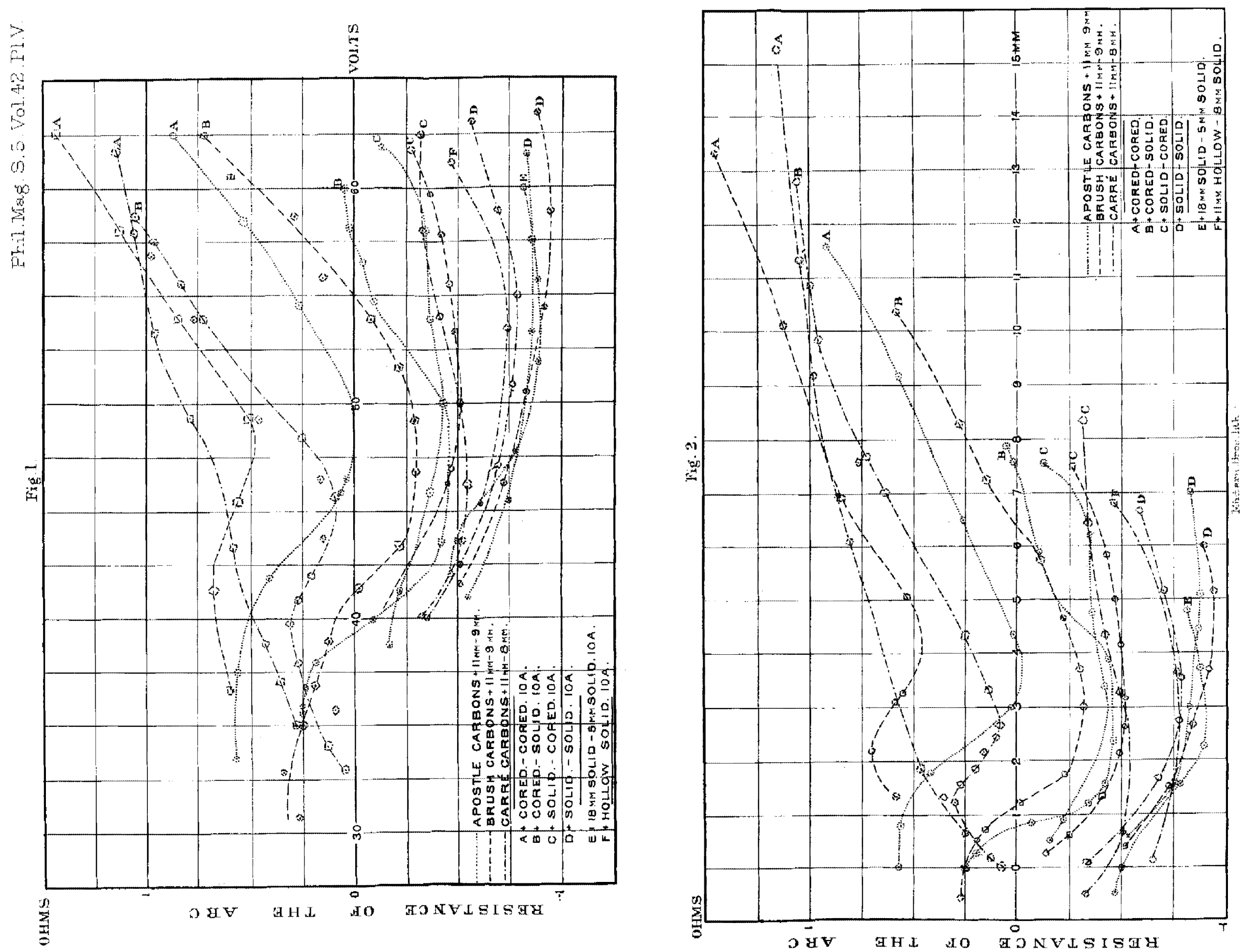
from our cells. The sizes of the Apostle carbons were those used by Mrs. Ayrton in her experiments.

That the diameter of the carbons used does not have any great effect on the resistance of the arc for the same current and voltage is shown by the curves $\mathrm{D}$ and $\mathrm{E}, \mathrm{Pl}$. IV. and fig. 1 , $\mathrm{Pl}$.V. These are the curves for two solid Apostle carbons-one set $\mathrm{D}$ having carbons 11 millim. and 9 millim. and the other set E having carbons 18 millim. and $\tilde{\partial}$ millim, in diameter respectively, and it will be seen that they lie very close together.

On the curves each make of carbons is represented by a particular kind of line as explained in the figure, the Apostle carbons, for instance, are represented by a dotted line. Curves bearing the same letter refer to the same combination of carbons in the different makes. For each make there are four combinations :- + cored, - cored ; + cored, - solid ; + solid, -cored; + solid, - solid; represented respectively by the letters $\mathrm{A}, \mathrm{B}, \mathrm{C}$, and $\mathrm{D}$.

The general characteristics of these curves are that the ordinates of those for + solid - solid are always negative, and those for +cored - cored always positive, while the other curves all lie between these two extremes, those which have the positive carbon solid always being more negative than those which have the positive cored. The greatest uniformity is met with in the case of the + solid - solid combination, in which all the curves lie close together. For this reason the curves for the Thomson-Houston carbons have been omitted, as they lie completely on the other curves, and would tend to cause confusion. In the case of cored carbons, however, the uniformity is not so marked, as the material of the core varies largely with the make. In fact, in a single carbon the core may vary considerably, and for this reason the curves for cored carbons are more troublesome to iake.

Pl. IV. shows the relation between the resistance of the are and the current that the arc is taking, the voltage at the curbons being kept constant. It will be noticed that in the case of those combinatious which have a solid positive, the number expressing the negative resistance of the are increases as the current decreases. With the other two combinationsthose having a cored positive-the curves for Brush and Carré carbons bave the same general characteristics, while those for the Apostle carbons cut these latter. This is probably due to peculiarities in the coring of the Apostle carbons.

$\mathrm{Pl}$. V. fig. 1 shows the relation between the resistance of the arc and the P.D. between the carbons, the current being kept constant at 10 umperes in all cases. These curves are more 
instructive than those in Pl. IV., as the character of the arc alters more with change of voltage than with change of current. As before, the curves for the solid carbons are all very close together. In this case they all show a minimum (maximum negative) value at about 55 volts. With combinations baving a cored positive, this minimum becomes more strongly marked and occurs at a lower voltage.

It has been our practice to examine the image of the arc on the screen and note when any change in the appearance of the arc takes place. It was first noticed by Mrs. Ayrton that with cored carbons under certain conditions a dark space appeared somewhere near the centre of the arc, dividing the purple glow into two parts. This was never noticed in the case of solid carbons. Our observations have fully confirmed this, and, moreover, we find that the dark space, when only one carbon is cored, is nearer this carbon. We have noted the appearance or disappearance of the dark space, and we find a remarkable coincidence between this point and the minimum of the curves. In the curves for cored carbons in Pl. V., to the right of the minimum point the arc always shows a dark space which becomes less marked on approaching the minimum, and at the minimum finally disappears. To the left of this point, althongh the dark space has quite disappeared, yet the difference in colour between the two parts of the glow is still observable, that near the negative carbon being of a redder tint than that near the positive.

PI.V. fig. 2 shows the relation between the length of the arc and its resistance at constant current. The general characteristics of these curves are the same as those of fig. 1, since the length of the are is roughly proportional to the voltage.

\section{Effect of the Core.}

Since the presence of a core in either carbon has such a marked influence on the resistance of the arc, we tried the effect of boring out the core of a Carré carbon. The result of using this hollow carbon as the positive is shown by curve $\mathrm{F}$ in PI. V. It will be seen that this curve follows very closely that obtained with both carbons solid. The effect was now tried of fillng this hollow carbon first with plaster of Paris, and then with kaolin. In both cases the resistance of the arc so produced was positive. Another effect of these cores was to cause the are to burn at a much lower voltage for a given current and arc length. The arc was, however, too unsteady to allow of a curve being taken. 
Particulurs of Cores.

The diameters of the various cores are as follows :-

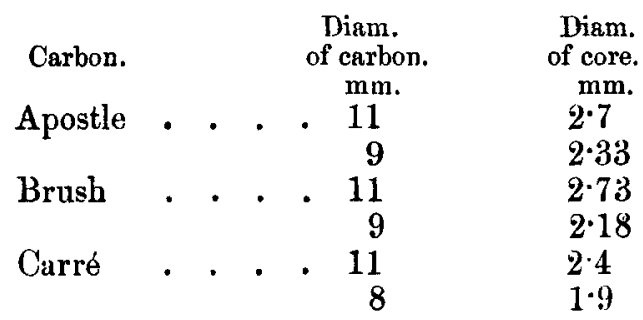

Inverted Arcs.

In the experiments hitherto described the positive carbon has always been uppermost. Experiments were now made to ascertain if the resistance of the arc is dependent upon whether the positive carbon is the upper or the lower.

Using solid carbons, the resistance is not appreciably altered by inverting the arc. With cored carbons, however, the resistance of the arc is altered, and moreover the physical character of the arc is changed. As we have pointed out above, the distinguishing feature of the are when using cored carbons is the appearance of a dark space situated near the centre of the purple glow, which appears to considerably increase the resistance of the arc. On inverting such an are, however, we find that this dark space completely disappears, and, as we should have expected, the resistance is considerably diminished. At low currents or P.Ds., the difference between the resistances of ordinary and inverted arcs diminishes till the two values become nearly equal. These are the conditions under which the dark space ordinarily disappears. Hence when the dark space has disappeared, inverting the are makes but little difference in its resistance, which is also the case with arcs using solid carbons, in which there is no dark space.

By placing the carbons horizontal, a value for the resistance of the arc can be obtained which is intermediate between the two values obtained by placing the positive carbon respectively above and beneath the negative carbon, the current and P.D. being the same in the three cases.

From consideration of the part played by the dark space, it would appear that the resistance of the arc is greatly affected by the state of the contact between the purple glow and the 
negative carbon. When the inside purple glow is in good contact with the negative carbon, the resistance of the arc is most negative. When, however, there is a dark space (caused by the presence of foreign matter or of carbon in a different physical state), and hence a lack of contact between this glow and the negative carbon, the resistance becomes increased.

\section{Hissing Arcs.}

An attempt was made, using Method I.; to find the resistance of a direct-current hissing are produced with accumulators, but it was found that even with the alternator at rest there w'as a large deflexion of the electrometer, showing that the current throngh a hissing are is oscillatory. In order to determine the frequency of the oscillation, a condenser and telephone were put across the arc. The sound beard in the telephone was, however, of no definite pitch.

A method suggested by Mr. Campbell was used to measure the amount of this oscillatory current. The current taken by the bissing arc was passed through the primary of a transformer, the secondary of which was put in series with a reflecting twisted-strip ammeter, the deflexion being noted. The arc lamp was then disconnected, and a known alternating current was sent through the primary, of such magnitude that the deflexion was the same as before. This known current has the same R.M.S. as the oscillatory current passing through the arc, for if the impedance of the secondary is large compared with its resistance, the deflexion of the ammeter is the same for the same R.M.S. current whatever the frequency. The arc was run at 14.5 amperes and 35 volts, Apostle carbons $+9 \mathrm{~mm}$. $-9 \mathrm{~mm}$. being used. While the arc was burning the deflexion of the ammeter varied hetween 200 and 250 scale-divisions. Primary currents of 0.43 ampere and 0.53 ampere, as measured by another twisted-strip ammeter, produced these scale readings respectively. Hence in this case the R.M.S. of the oscillatory current amounted to about 3 per cent. of the continuous current.

\section{Low Frequencies.}

On comparing our results with the steady $\frac{d \mathrm{~V}}{d \mathrm{~A}}$ taken from the slope of the curves published by Mrs. Ayrton, the agreement as to sign, which held in the case of solid carbons, was found to break down in the case of cored carbons; for while the general shape of the curves obtained by Mrs. Ayrton was the same for cored as for solid carbons, that is both curves 
except for short arc-lengths indicated a negative resistance*, we find that with both carbons cored the resistance is always positive. With a view to elucidating this point, we repeated Mr. Mather's experiments using both carbons cored, and found the $\frac{\delta \mathrm{V}}{\delta \mathrm{A}}$ negative as shown by the following table:-

$$
\begin{gathered}
\text { Brush Carbons. } \\
+11 \mathrm{~mm} .
\end{gathered}
$$

Arc taking 9 amperes at 43 volts.

\begin{tabular}{|c|c|c|c|c|}
\hline $\begin{array}{l}\text { Resistance } \\
\text { introduced. }\end{array}$ & Volts. & $\delta \mathrm{V}$ & Amperes. & $8 \mathrm{~A}$. \\
\hline$+\cdot 5$ & $44 \cdot 8$ & +1.8 & $8 \cdot 10$ & -.90 \\
\hline$+\cdot 4$ & $44 \cdot 2$ & $+1 \cdot 2$ & $8 \cdot 30$ & $-\cdot 70$ \\
\hline$+\cdot 3$ & 44 & $+1 \cdot 0$ & $8 \cdot 45$ & -.55 \\
\hline$+\cdot 2$ & $43 \cdot 7$ & +.7 & $8 \cdot 62$ & $-\cdot 38$ \\
\hline$+\cdot 1$ & $43 \cdot 3$ & $+\cdot 3$ & $8 \cdot 75$ & -.25 \\
\hline$-\cdot 1$ & $42 \cdot 8$ & $-\cdot 2$ & $9 \cdot 20$ & +20 \\
\hline$-\cdot 2$ & $42 \cdot 4$ & $-\cdot 6$ & $9 \cdot 38$ & +38 \\
\hline$-\cdot 3$ & $42 \cdot 0$ & $-1 \cdot 0$ & $9 \cdot 60$ & $+\cdot 60$ \\
\hline$-\cdot 4$ & $41 \cdot 6$ & $-1 \cdot 4$ & $9 \cdot 85$ & +85 \\
\hline$-\cdot 5$ & $41 \cdot 1$ & $-\overline{1} \cdot \overline{9}$ & $10 \cdot 08$ & +1.08 \\
\hline
\end{tabular}
Resistance in cireuit about 5 ohms.

In the curves connecting the P.D. between the carbons and the current, each point is obtained by taking the readings of ammeter and voltmeter after the arc has been made to burn for a very considerable time at constant current and arc-length, so that one may say that the "frequency" is zero. In this case, the resistance, if deduced from the slope of these curves, would be negative both for cored and for solid carbons. In Prof. Ayrton's original method, although the readings are taken as quickly as possible, still the "frequency" is small compared with the lowest frequency we have hitherto used, and both the carbons and the are may have time to alter before the ammeter and voltmeter can be read. 'This method would lead to the same conclusion as before, viz. that the resistance of the arc is always negative. Our measurements, however, with frequencies as low as $7 \cdot 5$ gave a positive resistance for arcs using cored carbons. Hence it seems that there must be a critical frequency, above which the resistance has a positive value which is independent of the frequency, and below which it appears to have a negative value, and further, that this frequency must lie between $7 \cdot 5$ and 0 .

* Mrs. Ayrton does indeed rind that for cored carbons at short lengths the curves slope slightly upwards, thus indicating a positive resistance.

Phil. Mag. S. 5. Vol. 42. No. 258. Nov. 1896. $2 \mathrm{H}$ 
Our former methods were not suitable for working with a frequency lower than 7, for at this frequency the needles of the voltmeter and ammeter began to vibrate with the alternating current. This vibration of the needles was made use of in the following way, to indicate the sign of the $\frac{d \mathrm{~V}}{d \mathrm{~A}}$ at frequencies lower than 7. The Gramme alternator used in the previous method was run as a rotatory transformer. By adjusting the continuous current passing through this transformer, and hence its speed, any desired frequency could be obtained down to as low as one complete alternation in 2 seconds. By an arrangement of mirrors the needles and scales of both ammeter and voltmeter could be observed simultaneously. In this way it could be seen whether the two needles were at any instant vibrating in the same or in opposite directions. It is evident that if the needles are vibrating in phase, that is if an increase of P.D. is accompanied by an increase of current, then $\frac{d \mathrm{~V}}{d \mathrm{~A}}$ must be positive; while if they are vibrating out of phase, that is if an increase of P.D. is accompanied by a decrease of current, then it is negative. With this arrangement, solid carbons at any frequency gave a negative resistance. With cored carbons, however, we found as we expected, that above a certain frequency the resistance was positive, while below this frequency the method gave a negative value for $\frac{d V}{d \mathrm{~A}}$. The critical frequency was found by observing the point at which the swing of the needles was uncertain, being sometimes in phase and sometimes out of phase. At this point the frequency was $1 \cdot 8$, at 1.9 the sign was certainly positive, and at 1.7 was negative. This result was obtained with all arcs whose resistance as given by our curves was positive (with one exception mentioned below), and was not obtained with any whose resistance was negative. In the case of arcs whose curves cross the zero line, the transition from positive to negative, as shown by the disappearance of the critical frequency, was well shown and corresponded with the crossing of the zero line. As an example, we ran an arc with Brush carbons $+11 \mathrm{~mm}$. cored $-9 \mathrm{~mm}$. solid at 10 amperes, the curve for which is marked B in fig. 1, Pl. V. It will be noticed that this curve crosses the zero line twice, at 55 volts and again at 41.3 volts : at 60 volts we found the sign of the $\frac{d \mathrm{~A}}{d \overline{\mathrm{V}}}$, as shown by the swing of the needles, to be positive at frequencies 
above 1.8 and negative below this frequency, at 45 volts it was negative at all frequencies; at 35 volts, however, it was positive at frequencies above $1 \cdot 8$, and also at frequencies below $1 \cdot 8$. That the resistance would be positive at all frequencies seemed probable, since the curves connecting the P.D. and current at constant arc-length for such carbons show that with small arc-lengths an increase of P.D. is accompanied by an increase of current.

The experiments have been carried out in the Physical Laboratory of the Central Technical College, and our best thanks are due to Professor Ayrton and Mr. Mather for much kind advice during the course of the work.

\section{Note.-Fluctuation of the Current given by a Continuous- Current Dynamo.}

An attempt was made to replace the cells in Method I. by a dynamo, but it was found that even with the alternator at resi the electrostatic voltmeter showed a large deflexion. This was evidently due to the oscillation of the current owing to the commutator of the dynamo having a finite number of segments. The oscillation was found to increase as the brushes were moved out of the sparkless position. The dynamo was a 5 kilowatt 2-pole machine, and was giving 10 amperes at 70 volts. By adjusting the brushes to the sparkless position this oscillatory current, measured in the same way as in the case of the hissing are above, could not be reduced below 2.5 per cent. of the continuous current, while by rocking the brushes out of this position it could be increased to as much as 9 per cent. without the sparking at the brushes being excessive. The commutator was in good condition. This oscillation may introduce errors in the measurement by polarized instruments of the currents given by dynamos owing to the differences between the mean and the R.M.S. of such a current.

\section{Microscopic Vision. By G. Johnstone Stoney, M.A., D.Sc., F.R.S.}

[Continued from p. 349.]

\section{Addition to Parm $I$.}

TThe following section should come into Part I. after Theorem 1, between $\$ \$ 7$ and 8.]

7 (a). Extension of Proposition 1.-In the proof of Proposition 1 it is assumed that the light emanates from each individual point of the object in an uninterrupted train of $2 \mathrm{H} 2$ 